\title{
Derechos humanos y empresas transnacionales: las debilidades del tercer pilar derivadas de las normas de promoción de inversiones. El caso Chevron como paradigma de la necesidad del Binding Treaty
}

\section{Human rights and transnational corporations: the weaknesses of the third pillar caused by investment promotion agreements. The Chevron case as a paradigm of the need for a Binding Treaty}

\author{
Adoración Guamán Hernández \\ Departament de Dret del Treball \\ Facultat de Dret \\ Universitat de València
}

\section{RESUMEN.}

El texto se centra en la necesidad de desarrollar normas internacionales vinculantes que aseguren el respeto por parte de las empresas transnacionales respecto de los derechos humanos, que pongan fin a la impunidad de estos actores económicos, así como para garantizar el acceso a la justicia para las víctimas y la reparación de las violaciones. Al analizar el caso de Chevron, el texto refleja que, si bien existen diferentes iniciativas a nivel internacional destinadas a alentar a las empresas transnacionales a comportarse de manera respetuosa con los derechos humanos, estas iniciativas siguen siendo insuficientes, ya sea por su falta de obligatoriedad o por su carácter parcial, dado que siguen dependiendo de la voluntad de estos actores económicos transnacionales. El caso Chevron y los laudos recaídos a lo largo del proceso arbitral de la empresa contra la República del Ecuador, muestran una paradoja alarmante: ciertos derechos, como el acceso a la justicia, diseñados para proteger los derechos humanos en su conjunto se están utilizando para salvaguardar los beneficios de determinadas empresas trasnacionales. Este caso, junto con muchos otros crímenes corporativos, apoya la idea de que el proceso de la Resolución 26/9, el llamado Binding Treaty, es actualmente el único que puede garantizar el acceso a la justicia y los recursos para las víctimas.

PALABRAS CLAVE.

Chevron, tratado bilateral de inversión, acceso a la justicia, mecanismos de reparación, Tercer Pilar de los Principios Rectores, derechos humanos, Binding Treaty.

\section{ABSTRACT.}

The text is focus on the need to develop internationally binding standards on transnational corporations and human rights in order to bring their impunity to an end, as well as ensuring access to justice for the victims and the reparation of the consequences. By analysing the Chevron case, the text reflects that although there are different initiatives at international level aimed at encouraging transnational companies to behave respectfully, these initiatives continue to be insufficient, either because of their lack of obligation or because of their partial nature, dependent on the voluntariness of these transnational economic actors. The Chevron case and the arbitration awards adopted during the arbitration proceedings against the Republic of Ecuador show an alarming paradox: certain rights, such as access to justice, designed to protect human rights as a whole, are being used to full effect to safeguard the profits of economic entities. This case, together with many other crimes, supports the idea that the process of Resolution 26/9, the so called Binding Treaty, is currently the only one potentially capable of ensuring the access to justice and remedies to victims.

KEY WORDS.

Chevron, bilateral investment treaty, access to justice, redress mechanisms, Third Pillar of the Guiding Principles, Human Rights, Binding Treaty.

CEFD 


\section{Introducción}

Desde la década de los años setenta del siglo pasado, la necesidad de controlar la actuación de las empresas transnacionales para asegurar la protección y el necesario respeto de los derechos humanos y de la naturaleza ha estado presente en los debates políticos, jurídicos y sociales, transitando muy lentamente a la esfera normativa. A lo largo de más de medio siglo de debates, en un escenario marcado por las constantes denuncias de violaciones de derechos humanos cometidas por estos actores económicos, se han planteado múltiples principios y resoluciones. Sin embargo, hasta el momento, no existe un marco jurídico común que, en el plano del derecho internacional de los derechos humanos, regule la obligación de respeto de las empresas respecto de los derechos humanos y garantice el acceso a la justicia y a la reparación de las víctimas.

La persistencia, incluso el agravamiento, de los obstáculos a la garantía y la reparación de los derechos humanos cuando son las empresas las que los violan (en particular las empresas con actividad transnacional) deriva en la actualidad de dos elementos intrínsecamente vinculados: por un lado, las permanentes mutaciones en la estructura, organización, estrategia y movilidad de los actores económicos que les permiten elegir los marcos normativos más propicios y eludir las jurisdicciones menos favorables; por otro, los fallos y carencias de marcos normativos existentes en el ámbito nacional e internacional para garantizar el disfrute y protección de los derechos humanos y su compleja relación con otras ramas del ordenamiento jurídico, en particular aquellas que protegen los derechos de las empresas en su categoría de "inversor extranjero" ${ }^{1}$.

Así, la experiencia demuestra que los actuales marcos regulatorios permiten, por acción u omisión, que las empresas transnacionales evadan su responsabilidad jurídica respecto de las violaciones de derechos humanos cometidas directamente o a través de la actividad desarrollada a lo largo de sus cadenas globales de producción. Estamos por tanto ante una situación de especial infradesarrollo del llamado

\footnotetext{
${ }^{1}$ Para un desarrollo de estas cuestiones se remite a textos fundamentales como son: DEVA, S., BILCHITZ, D. (Eds.); Building a Treaty on Business and Human Rights. Context and Contours. Cambridge. Cambridge University Press. 2017. DEVA, S., BILCHITZ, D., (Eds.); Human Rights Obligations of Business: Beyond the Corporate Responsibility to Respect?. Cambridge. Cambridge University Press. 2013; LYON-CAEN, A., SACHS, T., "The Responsibility of Multinational Entreprise. A constitutionalization process", en ROBÉ, J.-P., LYON-CAEN, A., VERNAC, S., (dir.), Multinationals and the constitutionalisation of the World Power System, Routlege, 2016;

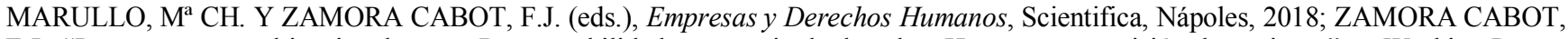
F.J., "Las empresas multinacionales y su Responsabilidad en materia de derechos Humanos: una visión de conjunto" en Working Papers "El Tiempo de los Derechos", No6, 2013; CANTÚ, H. (Coord.); Derechos Humanos y Empresas: Reflexiones desde América Latina. Costa Rica. Instituto Interamericano de Derechos Humanos, 2017; HERNÁNDEZ ZUBIZARRETA, J.; Las empresas transnacionales frente a los derechos humanos. Historia de una asimetría normativa. Vitoria, Egoa. 2009.
}

CEED Número 39 Publicación actas Congreso Internacional 70 Aniversario Declaración Universal de Derechos 
"Tercer Pilar" de los Principios Rectores ${ }^{2}$, principal instrumento actual vigente (y no vinculante) en el ámbito de Naciones Unidas respecto de la relación entre empresas y derechos humanos. Esta carencia y la persistencia de violaciones que quedan impunes y de víctimas indefensas está en la base de la propuesta del Binding Treaty (Instrumento Internacional Jurídicamente Vinculante sobre Empresas Transnacionales y otras empresas y los Derechos Humanos) que se está elaborando en el grupo de trabajo creado por la Resolución 26/9³ en el Consejo de Derechos Humanos de Naciones Unidas.

El presente artículo se enfoca en el análisis de los obstáculos al acceso a los mecanismos de garantía y reparación de los derechos humanos en casos de violación de los mismos por empresas transnacionales a través del estudio de un caso paradigmático, el caso Chevron.

Desde el año 1962 y durante casi 30 años, hasta 1992, la empresa petrolera Chevron, entonces Texaco, extrajo petróleo de la Amazonía ecuatoriana. Aquellas operaciones se caracterizaron por una doble realidad: fueron extremadamente rentables para la empresa y generaron una contaminación sin precedentes, devastando casi medio millón de hectáreas de selva amazónica. Rentabilidad y catástrofe estuvieron vinculadas de manera inextricable en lo que se ha llegado a denominar el "Chernobyl ecuatoriano", una catástrofe mayor que la del Exxon Valdés ${ }^{4}$.

Las demandas de las víctimas contra la transnacional han dado lugar a procesos judiciales en distintas jurisdicciones nacionales, internacionales y foros regionales. En 1993, las víctimas demandaron en New York a Texaco Petroleum Company ${ }^{5}$ por daños ambientales. La utilización de la doctrina del foro

\footnotetext{
${ }^{2}$ Consejo de Derechos Humanos, A/C/17/31, Principios Rectores sobre las empresas y los derechos humanos: puesta en práctica del marco de las Naciones Unidas para "proteger, respetar y remediar". 27 de marzo de 2011. Para un comentario crítico de estos principios se remite a: ESTEVE, J.E., "Los Principios Rectores sobre las empresas transnacionales y los derechos humanos en el marco de las Naciones Unidas para «proteger, respetar y remediar»: ¿hacia la responsabilidad de las corporaciones o la complacencia institucional?", Anuario Español de derecho internacional, №27, 2011; ESTEVE, J.E., "Planes de Acción Nacional sobre empresas y derechos humanos: la imperiosa complementariedad con normas vinculantes. Referencia al plan español". Anuario español de derecho internacional, $\mathrm{N}^{\circ} 34$, 2018; y a los contenido y bibliografía citada en: GUAMÁN, A., GONZÁLEZ, G.; Empresas Transnacionales y Derechos Humanos, Albacete, Bomarzo, 2018.

3 Resolución A/HRC/RES/26/9 «Elaboración de un instrumento internacional jurídicamente vinculante sobre las empresas transnacionales y otras empresas con respecto a los derechos humanos»: adoptada con votación en el Consejo de derechos humanos $(\mathrm{CDH})$ de las Naciones Unidas el 26 de junio de 2014. Disponible en https://documents-ddsny.un.org/doc/UNDOC/GEN/G14/082/55/PDF/G1408255.pdf?OpenElement

${ }^{4}$ Es importante comparar ambos casos porque, como ha señalado la doctrina, la contaminación en la Amazonía fue mayor pero los daños no han recibido ni reparación ni indemnización, algo que sí ocurrió respecto del vertido en Alaska. Sobre esta cuestión, para un análisis general de la realidad de Chevron como entidad y sus actividades se remite a la obra: CECEÑA, A.E., ORNELAS, R. (Coords.); Chevron. Paradigma de la catástrofe civilizatoria, Siglo XXI, México, UNAM, Instituto de Investigaciones Económicas, 2017.

${ }^{5}$ El 07 de septiembre del año 2001 la Federal Trade Comission aprobó el acuerdo que permitió la fusión de Chevron Corp. y Texaco Inc. Desde entonces la compañía pasó a llamarse ChevronTexaco, para posteriormente cambiar su nombre nuevamente a Chevron.
}

CEED Número 39 Publicación actas Congreso Internacional 70 Aniversario Declaración Universal de Derechos 
de no conveniencia por los tribunales en Nueva York y la petición y compromiso explícito de la empresa de ser juzgada en Ecuador llevó a las víctimas a demandar a la empresa ante la jurisdicción donde se cometió el daño, obteniendo una sentencia favorable que devino firme en $2012^{6}$. Esta sentencia, que condena a la empresa a pago de una indemnización de 9.500 millones de dólares, no ha podido ser ejecutada todavía. Chevron no tiene activos en el Ecuador y las víctimas se han visto forzadas a perseguir la ejecución de la sentencia en otras jurisdicciones donde sus demandas están siendo rechazadas (Brasil o Argentina) o siguen pendientes (Canadá). Al tiempo que era demandada, Chevron contratacó y ha conseguido llevar a las víctimas ante la justicia de Estados Unidos ${ }^{7}$ y al Estado ecuatoriano ante varios procedimientos de arbitraje inversor-Estado (Chevron I, II, y III).

El caso evidencia cómo, aun considerándose probada la existencia de un crimen corporativo contra la naturaleza y los derechos humanos, la culpable, la empresa, mantiene su impunidad gracias a la combinación de carencias normativas, estrategias jurídicas, captura corporativa y los "nuevos derechos" concedidos a las empresas transnacionales, en particular, el acceso a la justicia.

Por cuestiones de extensión, dado que la parte más conocida del caso Chevron es la litigación ante los tribunales, de Estados Unidos y de Ecuador, el tercer epígrafe de este artículo se centrará en el análisis del proceso arbitral y muy especialmente del laudo recaído en agosto del año 2018.

\footnotetext{
6 Chevron interpuso un Recurso Extraordinario de Protección alegando que la sentencia en su contra violentaba sus derechos constitucionales en más de 100 ocasiones diferentes. Este Recurso fue finalmente desechado por la Corte Constitucional el pasado 10 de julio de 2018, poniendo un cierre definitivo al litigio en Ecuador. Para un detalle del caso, se remite a: Prieto, J., Espinoza, G., "A binding treaty on corporate responsibility: a global solution to address the problem of corporate impunity. Lessons learned from Aguinda vs Chevron", en HOMA Publica: International Journal on Human Rigths and Business, No2, 2017; Procuraduría General del Estado, Caso Chevron: Defensa del Ecuador frente al uso indebido del arbitraje de inversión, Quito, 2015. Disponible en http://www.pge.gob.ec/images/stories/boletines/lanzamientolibrochevron/libro_Caso_CHEVRON.pdf; Ceceña, A.E., Ornelas, R. (Coords.) Chevron. Paradigma de la catástrofe civilizatoria, op.cit.

${ }^{7}$ El 1 de febrero de 2011, Chevron había presentado una demanda bajo la Ley de Organizaciones Corruptas e Influencia Extorsiva (RICO, por sus siglas en inglés) contra los demandantes ecuatorianos, sus abogados y representantes. La sentencia del caso RICO prohibió que los demandantes pudieran ejecutar su sentencia en contra de Chevron en Estados Unidos y que los abogados de los ecuatorianos se beneficiaran de cualquier modo (USDC, SDNY, Chevron Corporation v. Steven Donziger, et al., Case 1:11-cv-00691-LAK-JCF Document 1874. Filed 03/04/14; p. 469)
} 


\section{Derechos humanos y empresas transnacionales: los obstáculos al acceso a los remedios como} punto débil de la implementación de los Principios Rectores sobre empresas y derechos humanos (el Tercer Pilar)

Como es bien sabido, el acceso a los mecanismos de reparación ante una violación de los derechos humanos es un requisito fundamental sin el cual no puede considerarse la existencia jurídica completa de los mismos. Así, los instrumentos de reparación, en particular los de carácter judicial, pero también los extrajudiciales como complemento a los primeros, son un elemento esencial de la plasmación jurídica de los derechos humanos.

La afirmación de este carácter imprescindible de los instrumentos de garantía y reparación se revela especialmente importante cuando transitamos del ámbito relacional Estado-derechos humanos y nos encontramos en el contexto empresas-derechos humanos. Los mecanismos de garantía y reparación tradicionalmente estructurados y pensados para proteger los derechos humanos del individuo y los grupos en los que se integra frente a violaciones cometidas por el Estado se revelan inadecuados o incluso se afirman inaplicables cuando el actor que comete la violación es una persona jurídica.

La necesidad de implementar estos mecanismos es uno de los grandes temas que rodea el debate en torno a la eficacia de los "Principios Rectores sobre las empresas y los derechos humanos: puesta en práctica del marco de las Naciones Unidas para "proteger, respetar y remediar"8. Estos Principios Rectores no establecen nuevas obligaciones de derecho internacional, ni instauran un mecanismo coercitivo para asegurar su cumplimiento ni establecen obligaciones directas para las empresas. Los mismos se basan en el reconocimiento de tres grupos de principios fundamentales, los denominados "tres pilares", que son los siguientes: las obligaciones de los Estados de respetar, proteger y cumplir los derechos humanos y las libertades fundamentales (Primer Pilar); el papel de las empresas, que deben cumplir todas las leyes aplicables y respetar los derechos humanos (Segundo Pilar); y la necesidad de

\footnotetext{
${ }^{8}$ Consejo de Derechos Humanos, A/C/17/31, Principios Rectores sobre las empresas y los derechos humanos: puesta en práctica del marco de las Naciones Unidas para "proteger, respetar y remediar". 27 de marzo de 2011. Este documento contiene 31 principios ordenados en tres pilares y divididos entre principios fundacionales y operacionales. Los Principios deben ser desarrollados por los llamados "Planes Nacionales de Actuación".
} 
que los derechos y obligaciones vayan acompañados de recursos adecuados y efectivos en caso de incumplimiento (Tercer Pilar) ${ }^{9}$.

Como ha recordado el Grupo de Trabajo sobre la cuestión de los derechos humanos y las empresas transnacionales y otras empresas "el derecho a una reparación efectiva por los daños causados es un principio fundamental del derecho internacional de los derechos humanos" y el acceso a una reparación efectiva es un componente esencial de los Principios Rectores ${ }^{10}$. Más aún, se ha llegado a afirmar que “el acceso a la reparación por la vía judicial es la columna vertebral del tercer pilar de los Principios Rectores"11. A pesar de este reconocimiento, es también una opinión ampliamente compartida que el Tercer Pilar en general y el concreto acceso a los mecanismos judiciales en particular, están recibiendo un desarrollo muy desigual y que los obstáculos existentes para la reparación de las víctimas no se han removido $^{12}$.

\subsection{Obstáculos para el acceso a los mecanismos de reparación en situaciones de violaciones de derechos humanos cometidas por empresas transnacionales.}

Transcurridos ocho años de la adopción de los Principios Ruggie, los obstáculos al acceso a la tutela judicial efectiva en particular y a la remediación en general se mantienen ${ }^{13}$ y sigue siendo una cuestión

\footnotetext{
${ }^{9}$ Del conjunto de los 6 Principios de este Pilar nos interesan dos, el 25 y el 26. En concreto, el Principio Fundacional 25 contiene la obligación de los Estados de tomar medidas apropiadas para garantizar, por las vías judiciales, administrativas, legislativas o de otro tipo que correspondan, que cuando se produzcan ese tipo de abusos en su territorio y/o jurisdicción los afectados puedan acceder a mecanismos de reparación eficaces. A continuación, el Principio Operativo 26 se dirige a los mecanismos judiciales estatales, para afirmar que los Estados deberían asegurar la eficacia de los mismos y limitar los obstáculos legales, prácticos y de otros tipos que puedan conducir a una denegación del acceso a los mecanismos de reparación. Debe recordarse además que, en el ámbito del Primer pilar, el Principio Operativo 8 recuerda que "los Estados deben mantener un marco normativo nacional adecuado para asegurar el cumplimiento de sus obligaciones de derechos humanos cuando concluyan acuerdos políticos sobre actividades empresariales con otros Estados o empresas, por ejemplo, a través de tratados o contratos de inversión". El comentario al Principio 25 señala con claridad que "si los Estados no adoptan las medidas necesarias para investigar, castigar y reparar las violaciones de los derechos humanos relacionadas con empresas cuando se producen, el deber de protección de los Estados puede debilitarse e incluso carecer de sentido"; mientras, ya respecto de los mecanismos judiciales, el comentario al Principio 26 señala que "los mecanismos judiciales eficaces son esenciales para garantizar el acceso a la reparación. Su capacidad para hacer frente a las violaciones de los derechos humanos relacionadas con empresas depende de su imparcialidad, integridad y capacidad de hacer respetar las debidas garantías procesales".

${ }^{10}$ Informe del Grupo de Trabajo sobre la cuestión de los derechos humanos y las empresas transnacionales y otras empresas, presentado de conformidad con las resoluciones 17/4 y 35/7 del Consejo de Derechos Humanos el 18 de julio de 2017 (A/72/162)

${ }^{11}$ Informe del Grupo de Trabajo sobre la Cuestión de los Derechos Humanos y las Empresas Transnacionales y Otras Empresas acerca del sexto período de sesiones del Foro sobre las Empresas y los Derechos Humanos. A/HRC/38/49

${ }^{12}$ Vid. en particular el informe: Skinner, G., et alt., «El Tercer Pilar: El acceso efectivo a los recursos judiciales para las víctimas de las violaciones de los derechos humanos cometidas por empresas transnacionales», International Corporate Accountability Roundtable, icar, core y European Coalition for Corporate Justice, ECCJ, 2013

${ }^{13}$ Entre los informes que contienen evidencias es este sentido podemos destacar los siguientes:
}

CEED Número 39 Publicación actas Congreso Internacional 70 Aniversario Declaración Universal de Derechos 
comúnmente aceptada que la mayoría de las víctimas de violaciones de derechos humanos cometidas por empresas transnacionales se han saldado o bien con la impunidad de la empresa transnacional implicada y la indefensión de las víctimas o bien con una reparación económica en mediación privada, evitando la actuación de los tribunales.

Las dificultades del tercer pilar no se ignoraban en el momento de redacción de los principios. De hecho, el comentario del Principio 26 enumeró con claridad los obstáculos legales que pueden impedir o limitar la eficacia de los mecanismos judiciales.

- Informe del Alto Comisionado de las Naciones Unidas para los Derechos Humanos, titulado "Mejorar la rendición de cuentas y el acceso a las reparaciones para las víctimas de violaciones de los derechos humanos relacionadas con actividades empresariales", A/HRC/32/19, de 16 de mayo de 2016. El punto de partida de este informe fue la constatación, ya en 2013, de la necesidad de reforzar el Tercer Pilar que llevó al Alto Comisionado como parte de su mandato de impulsar la protección y promoción de los derechos humanos en todo el mundo, a iniciar un proceso destinado a ayudar a los Estados a fortalecer su aplicación de este tercer pilar, especialmente en casos de violaciones graves de derechos humanos relacionadas con actividades empresariales. Entre otras cuestiones el informe señaló que: “"Las consecuencias de las actividades de las empresas sobre los derechos humanos dan lugar a la apertura de causas en muchas jurisdicciones, pero las demandas privadas suelen finalizar sin que se llegue a juicio y, en los casos en que se obtiene una reparación, a menudo esta no satisface la norma internacional en materia de reparación adecuada, efectiva y rápida del daño sufrido".

- Informe del Grupo de Trabajo sobre la cuestión de los derechos humanos y las empresas transnacionales y otras empresas, $\mathrm{A} / \mathrm{HRC} / 35 / 33$, "Mejores prácticas y forma de aumentar la eficacia de la cooperación transfronteriza entre los Estados en lo que respecta a la aplicación de la legislación en la cuestión de las empresas y los derechos humanos” (abril, 2017): “ (...) las organizaciones de la sociedad civil han tratado infructuosamente de interponer demandas ante las fiscalías nacionales. Los Estados siguen mostrando falta de voluntad politica para hacer frente a las violaciones de los derechos humanos relacionadas con las actividades empresariales desde la perspectiva del derecho penal".

- Informe del Grupo de Trabajo sobre la cuestión de los derechos humanos y las empresas transnacionales y otras empresas, presentado de conformidad con las resoluciones 17/4 y 35/7 del Consejo de Derechos Humanos el 18 de julio de 2017 (A/72/162). $\mathrm{Su}$ recomendación general consistía en que los Estados debían establecer mecanismos eficaces de reparación, tanto judiciales como extrajudiciales, y eliminar los obstáculos al acceso a esos mecanismos; los titulares de derechos debían ocupar un lugar central en el proceso de reparación, y había de prestarse una atención debida a sus diversas experiencias, en particular por lo que se refiere a la importancia capital de adoptar una perspectiva de género; debía aspirarse a la libertad con respecto al miedo a la victimización y a los ataques dirigidos contra defensores de los derechos humanos; y las labores de reparación debían ser eficaces, en cuanto al proceso en sí y a los resultados

- Informe del Grupo de Trabajo sobre la Cuestión de los Derechos Humanos y las Empresas Transnacionales y Otras Empresas acerca del sexto período de sesiones del Foro sobre las Empresas y los Derechos Humanos. A/HRC/38/49, que señala las carencias en el ámbito del acceso a la investigación, rendición de cuentas y reparación.

- Informe de la sexta sesión del Foro de Empresas y Derechos Humanos de 2018, A/HRC/38/49, donde se indicaban las carencias en el ámbito del acceso a la investigación, rendición de cuentas y reparación, enfatizando, entre otras cuestiones, que "los regímenes jurídicos aplicables al respeto de los derechos humanos por parte de las empresas no están por lo general concebidos desde la óptica de los derechos humanos, lo cual genera problemas, como ocurre con los resultados cuando las únicas sanciones aplicables (como una multa) tal vez no se ajusten a la gravedad del abuso o el daño provocado".

- Estudio encargado por la Oficina del Alto Comisionado de Naciones Unidas para los Derechos Humanos, titulado "Corporate liability for gross human rights abuses. Towards a fairer and more effective system of domestic law remedies", realizado por Jennifer Zerk en el año 2014. La autora del estudio enumera hasta 22 casos de violaciones graves de derechos humanos cometidas por empresas. Ninguno de los casos que ya han sido cerrados ha finalizado con una sentencia condenatoria a una empresa por la comisión de violaciones a derechos humanos. Un elevado porcentaje se acabó solucionando por negociación extrajudicial entre las $\begin{array}{lllll}\text { víctimas } & \mathrm{y} & \text { las } & \text { empresas. } & \text { Disponible }\end{array}$ https://www.ohchr.org/Documents/Issues/Business/DomesticLawRemedies/StudyDomesticeLawRemedies.pdf

CEFD Número 39 Publicación actas Congreso Internacional 70 Aniversario Declaración Universal de Derechos 
Se trata de una afirmación también por la doctrina especializada, entre la que destaca el informe realizado por los profesores Skinner, McCorquodale y De Schutter en $2013^{14}$ divide los obstáculos al acceso a la reparación en los siguientes grandes apartados: presentación de demandas por daños causados en el extranjero; la doctrina del forum non conveniens; la responsabilidad de las empresas por violaciones de los derechos humanos; la prescripción para la presentación de demandas; las inmunidades y las doctrinas de no justiciabilidad; la determinación del derecho aplicable; la actividad probatoria en las violaciones de los derechos humanos; el costo de los litigios transnacionales. La mayoría de estos obstáculos aparecen de manera clara en la primera fase judicial del Caso Chevron, el llamado "Caso Aguinda" o el proceso de las víctimas contra la empresa ante la jurisdicción norteamericana.

A estos obstáculos alacceso a la reparación efectiva, en su vertiente concreta del acceso a la justicia, se suman otras, especialmente relevantes en los últimos años: el contenido de los acuerdos de inversión y su sistema de solución de controversias inversor-Estado (ISDS). Estos obstáculos, a los que se dedica el resto del artículo, se ejemplifican claramente en las demandas de arbitraje de inversiones interpuestas por Chevron contra el Ecuador.

\subsection{El uso de las normas de atracción y protección de la inversión extranjera como vía para garantizar la impunidad de las empresas transnacionales y obstaculizar el acceso a la justicia de las víctimas.}

Resumiendo lo señalado en el apartado anterior, es posible afirmar que la obligación de proteger los derechos humanos implica que los Estados deberán garantizar que todas las personas dispongan de recursos accesibles y efectivos para reivindicar esos derechos y que se establezcan en el derecho interno mecanismos judiciales y administrativos adecuados para conocer de las quejas sobre violaciones de los derechos ${ }^{15}$. Esta obligación en ocasiones colisiona directamente con la utilización por parte de las empresas de los mecanismos de resolución de controversias entre inversores extranjeros y Estados.

\footnotetext{
${ }^{14}$ Skinner, G., et alt.; El Tercer Pilar: El acceso efectivo a los recursos..., op. cit.

15 Observación General No. 31 al PIDCP, Naciones Unidas, Comité de derechos humanos, Observación General No. 31: Naturaleza de la obligación jurídica general impuesta a los Estados Partes en el Pacto, Doc. ONU CCPR/C/21/Rev.1/Add.13, 26 de mayo de 2004.
}

CEED Número 39 Publicación actas Congreso Internacional 70 Aniversario Declaración Universal de Derechos 
Un tratado bilateral de inversión (TBI) puede definirse como un acuerdo entre dos Estados para instituir un marco estable que promueva y encuadre normativamente los flujos de inversión que se desarrollan entre ellos ${ }^{16}$. Los TBI (denominados en también “acuerdos de protección y promoción de inversiones") regulan dos diferentes tipos de mecanismos de resolución de controversias mediante el arbitraje. Por un lado, los mecanismos de solución de controversias Estado-Estado y, por otro lado, los mecanismos de resolución de controversias inversor-Estado (los clásicos ISDS).

Definiéndolo de manera sintética, podemos entender que el ISDS es un sistema que permite a los inversores extranjeros demandar a un Estado ante un tribunal privado de arbitraje y eludir el sistema judicial estatal ${ }^{17}$. En la actualidad, este sistema está siendo criticado por cuestiones como: el elevado número de demandas ${ }^{18}$, la parcialidad de los árbitros, la opacidad del proceso o la utilización contra medidas gubernamentales como la revocación de licencias o las expropiaciones directas o indirectas ${ }^{19}$.

La existencia de incompatibilidades entre las normas de Derechos Humanos y los acuerdos de comercio e inversión ha encontrado respuesta en diversos Comentarios Generales, redactados en el marco del desarrollo de diversos instrumentos de derechos humanos del sistema de Naciones Unidas.

\footnotetext{
${ }^{16}$ Sobre esta cuestión se remite a, inter alia: SORNARAJAH, M., The international Law of foreing investment, Cambridge, Cambridge University Press, 2004; VICENTE BLANCO, D. J., "El conflicto histórico por el régimen de la inversión extranjera directa", en VICENTE BLANCO, D. J., (Ed.) La libertad del dinero, Valencia, Germanía, 2003; DOLZER, R. Y STEVENS, M., Bilateral Investment Treaties, Nijhoff, Dordrecht, 1995; EBERHARDT, P., "La protección de las inversiones en una encrucijada. La TTIP y el futuro del derecho global de las inversiones”, Friedrich-Ebert-Stiftung, Política Global y Desarrollo, Berlín, 2014.

${ }^{17}$ El inversor puede hacer uso de este sistema para proteger sus derechos de propiedad o los beneficios reales o previstos de su inversión frente a una amenaza a los mismos derivada bien de la aprobación de una norma estatal - relativa, por ejemplo, a la salud, medio ambiente o derechos laborales-, bien de la ejecución de una política social que pueda aminorar los beneficios de la inversión extranjera. Las características fundamentales del mecanismo, en su configuración habitual, serían las siguientes: ausencia de intervención del Estado de origen del inversor, que no tiene que autorizar o apoyar la demanda contra el Estado de recepción; libre elección del inversor entre demandar al Estado por el procedimiento judicial ordinario, como debe hacer cualquier empresa nacional, o acudir directamente al tribunal de arbitraje internacional; reconocimiento de un acceso exclusivo a los inversores extranjeros, que excluye a las empresas nacionales; falta de recursos o de procedimientos de revisión de las decisiones adoptadas por los árbitros; composición ad hoc de los tribunales de arbitraje para cada caso; confidencialidad del proceso y de los resultados; extensión del impacto del mecanismo más allá del tradicional control de las expropiaciones o nacionalizaciones y su afectación, también, sobre el conjunto de decisiones o normas que puedan adoptar los poderes públicos. KRAJEWSKI, M., Modalities for investment protection and Investor-State Dispute Settlement (ISDS) in TTIP from a trade union perspective, Friedrich-Alexander-Universität Erlangen-Nürnberg, 2015. Disponible en http://library.fes.de/pdf-files/bueros/bruessel/11044.pdf; EBERHARDT, P., Investment Protection at a Crossroads. The TTIP and the Future of International Investment Law. Friedrich Eber Stiftung, 2014. http://library.fes.de/pdf-files/iez/global/10875.pdf.

${ }^{18}$ Según los datos de la UNCTAD, en 2017 se iniciaron al menos 65 nuevos casos de SCIE basados en tratados, con lo que el número total de casos conocidos asciende a 855. A finales de 2017, los inversores habían ganado alrededor del $60 \%$ de todos los casos en los que se llegó a una decisión sobre el fondo de la cuestión. Vid. UNCTAD, Informe sobre las inversiones en el mundo, 2018. Vid. igualmente http://investmentpolicyhub.unctad.org/ISDS? status=1000

${ }^{19}$ Sobre esta cuestión se deriva al debate que se está produciendo en el ámbito de la UNCITRAL sobre la reforma del ISDS. Para un resumen del estado del mismo es útil la lectura del "Draft report of Working Group III (Investor-State Dispute Settlement Reform) on the work of its thirty-sixth session”, de noviembre de 2018 (A/CN.9/964) y en general los documentos del Working Group III, dedicado a la cuestión, y disponibles en: https://uncitral.un.org/en/working_groups/3/investor-state.
}

CEED Número 39 Publicación actas Congreso Internacional 70 Aniversario Declaración Universal de Derechos 
Como afirmación marco, los diversos informes destacan que los acuerdos de liberalización del comercio no deben restringir ni menoscabar la capacidad de un país de garantizar el pleno ejercicio de los derechos (al agua, a la salud, a la seguridad social) ${ }^{20}$. En concreto, el Profesor De Schutter, en su propuesta de Principios Rectores sobre el informe de impacto en los derechos humanos de los tratados de comercio e inversión, del año $2011^{21}$, señaló que:

- la obligación de respeto debe impedir que un Estado suscriba un acuerdo de comercio e inversión que le obligue a reducir la protección que otorga a determinados derechos;

- la obligación de proteger exige que los Estados no suscriban tratados que los incapaciten o que aminoren su capacidad para controlar y en su caso enjuiciar los comportamientos de los actores privados que vulneren los derechos humanos, lo que suele ocurrir cuando se suscriben acuerdos que integran los mecanismos de resolución de controversias inversor-estado (ISDS)

- la obligación de realizar implica la imposibilidad de que un Estado acepte una serie de obligaciones que le imposibiliten para el adecuado desarrollo de los derechos humanos.

Por su parte, el Relator De Zayas ha subrayado que "la gran mayoría de acuerdos de comercio y la totalidad de los acuerdos de inversión suponen una limitación respecto de la capacidad de los Estados firmantes de respetar, proteger y realizar los derechos humanos"22.

En el ámbito de la Unión Europea, la Comisión también ha resaltado la necesidad de mantener una coherencia entre el conjunto de políticas de la Unión. En este sentido, en el Plan de Acción de la UE

\footnotetext{
${ }^{20}$ Committee on Economic, Social and Cultural Rights, E/C.12/2002/11, General Comment No. 15 (2002): The right to water, paras 35 y 36. Committee on Economic, Social and Cultural Rights, E/C.12/GC/19, General Comment No. 19 (2008): The right to social security, paras. 54 y $57-58$.

${ }^{21}$ Asamblea General, A/HRC/19/59/Add.5, Report of the Special Rapporteur on the right to food, Olivier De Schutter, Addendum, Guiding principles on human rights impact assessments of trade and investment agreements, para. 1.

${ }^{22}$ Entre otras cuestiones, y enfocándose en los mecanismos de ISDS, De Zayas identificó como principales problemas los siguientes: la garantía a los inversores extranjeros de más derechos que a los inversores nacionales, con lo cual se establecen condiciones de competencia desigual; la falta de transparencia de los tribunales de solución de controversias entre inversores y Estados y las preocupaciones por la independencia y la imparcialidad de los árbitros; el conocido como efecto "Chilling Effect" que se deriva del temor a una posible sentencias condenatoria y que lleva a los Estados a abandonar medidas para proteger la salud pública y a reducir las exigencias de las normas ambientales, pudiendo disuadirles también en el futuro de tomar medidas para respetar, proteger y cumplir sus obligaciones en materia de derechos humanos, con el consiguiente efecto negativo en el orden internacional democrático y equitativo. Cuarto informe del Experto Independiente sobre la promoción de un orden internacional democrático y equitativo, Alfred-Maurice de Zayas, presentado de conformidad con lo dispuesto en la resolución 69/178. A/70/285, 5 de agosto de 2015.
} 
para los Derechos Humanos y la Democracia (2015-2019), se indica que la UE y sus Estados miembros se comprometen a emprender acciones para impulsar la cuestión de las empresas y los derechos humanos en el marco de los Principios Rectores, asegurando entre otras cuestiones la coherencia entre las políticas de derechos humanos y de comercio e inversión.

Por su parte, el Parlamento Europeo ha señalado en reiteradas ocasiones la necesidad de garantizar la compatibilidad entre la política comercial y las garantías de los Derechos Humanos, en este sentido, el conocido como Informe Corrao ${ }^{23}$ y muy en particular la Resolución del Parlamento Europeo, de 4 de octubre de 2018, sobre la contribución de la Unión a un instrumento vinculante de las Naciones Unidas sobre las empresas transnacionales y otras empresas con características transnacionales con respecto a los derechos humanos (2018/2763(RSP) ${ }^{24}$. Se trata, según el Parlamento, de una cuestión esencial para superar los desequilibrios de la globalización y para dar prioridad a los derechos de las personas y al planeta. El ejemplo paradigmático que demuestra lo acertado de esta afirmación del Parlamento reside en el caso Chevron.

\section{El arbitraje de inversiones entre Chevron y el Estado Ecuatoriano como ejemplo de vulneración del derecho a los mecanismos de reparación en los casos de violaciones de derechos humanos cometidos por empresas}

Chevron ha demandado al Ecuador en tres ocasiones (Chevron I en 2004; Chevron II en 2006 y Chevron III en 2009), en los tres casos, la utilización del mecanismo de arbitraje de inversiones ha respondido a una actuación defensiva de la empresa, persiguiendo el objetivo de obtener por la vía del arbitraje, y la protección que le otorga el derecho comercial, la impunidad frente a las condenas

${ }^{23}$ Informe sobre la responsabilidad de las empresas por violaciones graves de los derechos humanos en terceros países de 19 de julio de $2016(2015 / 2315$ (INI).

24 Resolución disponible en: http://www.europarl.europa.eu/sides/getDoc.do?pubRef=-//EP//TEXT+TA+P8-TA-20180382+0+DOC+XML+V0//EN\&language=EN. Entre otras cuestiones, el Parlamento reiteró en esta Resolución la primacía de los derechos humanos en el Derecho internacional: “"Considerando que existe una asimetría entre los derechos y las obligaciones de las empresas transnacionales, sobre todo en los tratados de protección de la inversión, en los que se conceden a los inversores amplios derechos, como el «trato justo y equitativo», que no siempre tienen el contrapeso de obligaciones vinculantes y ejecutorias en términos de respeto de los derechos humanos y la legislación laboral y medioambiental a lo largo de toda la cadena de suministro"; y la necesidad de consolidarla: "Mediante un sistema inequívoco por el que las obligaciones de derechos humanos prevalezcan efectivamente sobre otros tipos de obligaciones en conflicto y se dispongan mecanismos adecuados para hacer cumplir la legislación en materia de derechos humanos, de control y de reparación, junto con un sistema adecuado de sanciones y compensaciones en caso de violaciones". 
recaídas en la jurisdicción ordinaria ${ }^{25}$. El último laudo sobre este caso (Segundo Laudo Parcial de la Fase II, en adelante Laudo de $2018^{26}$ ), que decide sobre el fondo del asunto fue hecho público en agosto de 2018. Este laudo es el ejemplo perfecto de cómo el mecanismo de resolución de controversias inversor-Estado es utilizado para convertir décadas de litigio ante la jurisdicción ordinaria en un ejercicio inútil y para arrumbar el derecho humano fundamental al acceso a la justicia.

El caso Chevron III comenzó el 23 de septiembre de 2009 cuando las mismas empresas ${ }^{27}$ que protagonizaron Chevron I y II presentaron una nueva demanda de arbitraje basada en el TBI entre Ecuador y Estados Unidos. El objetivo fundamental de las demandantes en este asunto era la obtención de una actuación del Estado impidiendo la ejecución de una sentencia (la del caso Lago Agrio) que estaba todavía pendiente en el momento de interponer la demanda arbitral. Así, el caso Chevron III ${ }^{28}$ y sus diferentes laudos supone un salto cualitativo en cuanto al grado de afectación a la obligación de los Estados de proteger y garantizar los derechos humanos y consecuentemente al derecho fundamental al acceso a la justicia.

En el anuncio del arbitraje, presentado en septiembre del año 2009, Chevron afirmó que el Estado ecuatoriano había violado el TBI vigente en el momento de la demanda, entre Estados Unidos y Ecuador, quebrando sus obligaciones de proteger las inversiones que la empresa realizó en el país. A efectos de analizar esta afirmación deben tenerse en cuenta las cuestiones de admisibilidad y las razones de fondo alegadas por la empresa y la defensa del Estado.

\footnotetext{
${ }^{25}$ Para un análisis de este caso, hasta el año 2013, vid. PIGRAU, A., "The Texaco-Chevron case in Ecuador: Law and justice in the age of globalization”, Revista catalana de dret ambiental, Vol. V Núm. 1 (2014): 1 - 43.

${ }^{26}$ PCA CASE NO. 2009-23. In the matter of an arbitration before a tribunal constituted in accordance with the treaty between the United States of America and the Republic of Ecuador concerning the encouragement and reciprocal protection of investment, signed 27 august 1993 (the "treaty" or "BIT") and the UNCITRAL arbitration rules 1976 (the "UNCITRAL arbitration rules"). between: 1. Chevron Corporation ("Chevron") 2. Texaco Petroleum Company ("TexPet") (both of the United States of America) The First and Second Claimants - and - The Republic of Ecuador, the Respondent. Second Partial Award on Track II dated 30 August 2018. Disponible en: https:/www.italaw.com/sites/default/files/case-documents/italaw9934.pdf. En adelante: Laudo de 2018.

27 Chevron Corporation (“Chevron") y Texaco Petroleum Company (“TexPet”) presentan su notificación del inicio del procedimiento arbitral contra la República del Ecuador el 23 de septiembre de 2009. De ahora en adelante, como ya se ha señalado, se les denominará "las demandantes" o sencillamente Chevron.

${ }^{28}$ PCA CASE NO. 2009-23. In the matter of an arbitration before a tribunal constituted in accordance with the treaty between the United States of America and the Republic of Ecuador concerning the encouragement and reciprocal protection of investment, signed 27 august 1993 (the "treaty" or "BIT") and the UNCITRAL arbitration rules 1976 (the "UNCITRAL arbitration rules"). between: 1. Chevron Corporation ("Chevron") 2. Texaco Petroleum Company ("TexPet") (both of the United States of America) The First and Second Claimants - and - The Republic of Ecuador, the Respondent. Claimants Notice of Arbitration. Disponible en: https://www.italaw.com/sites/default/files/case-documents/ita0155_0.pdf.
} 
La admisibilidad del asunto y la competencia del panel de arbitraje, que implica la aceptación de la aplicabilidad del TBI ratione tempore y ratione materia, ha sido sin duda la cuestión más debatida. Debe tenerse en cuenta que el Tratado con Estados Unidos fue suscrito el 27 de agosto de 1993 y ratificado en octubre de 1995. Su entrada en vigor se produjo el 11 de mayo de 1997; sin embargo, la empresa, abandonó el territorio del Ecuador y finalizó su inversión en este país en el año 1992.

Para justificar la aplicación del TBI y la competencia del panel de arbitraje, la empresa afirmó que su inversión continuó en Ecuador durante los años posteriores a su retirada del país en base a los llamados Acuerdos de Liberación (1995, 1996 y 1998). En este contrato la empresa se comprometió a realizar un trabajo de reparación ambiental (mínimo) a cambio del cual el gobierno le garantizó una amplia impunidad respecto de sus acciones ${ }^{29}$, es decir, el ejecutivo se comprometía a no interponer ni directamente ni vía Petroecuador una demanda contra las empresas "exoneradas" 30.

\footnotetext{
${ }^{29}$ Debe cuestionarse el concepto de inversión que se utiliza para admitir la demanda y condenar al Estado. Las demandantes sostuvieron, para conseguir la admisión de la jurisdicción y la aplicabilidad del Tratado, que los gastos que la empresa realizó para ejecutar las medidas compensatorias y de remediación eran una inversión que entraría bajo la protección del TBI. Sin embargo, el concepto de inversión no puede abordar cualquier tipo de actuación de una empresa en un territorio de un Estado. Esto significaría que el TBI protege cualquier actuación de la empresa, lo que, evidentemente, vulnera el principio básico de seguridad jurídica respecto de las obligaciones a las que se compromete el Estado. Solo a modo de ejemplo es interesante señalar que las definiciones de inversión extranjera incluyen habitualmente los requisitos de "interés duradero", relación a largo plazo con una empresa estatal, influencia significativa sobre la gestión de la empresa en la que se invierte, etc... (Vid. Organización para la Cooperación y el Desarrollo Económicos (OCDE) OECD Benchmark Definition of Foreign Direct Investment, París, OCDE, 1996) Ninguna de estas características podía predicarse del Contrato de 1995, cuya única finalidad era realizar unas tareas de limpieza para recibir a cambio una amplia exoneración de responsabilidades.
}

30 “Contrato para la ejecución de trabajos de reparación medioambiental y liberación de obligaciones, responsabilidades y demandas, fue celebrado entre el Gobierno del Ecuador, Petroecuador y Texpet, el 4 de mayo de 1995”. El texto original de este contrato de 1995 puede encontrar adjunto en el Primer Laudo Arbitral Parcial de la Fase I, de 17 de septiembre de 2013, disponible https:/www.italaw.com/sites/default/files/case-documents/italaw1585.pdf. Para entender la amplitud de esta exoneración es fundamental la lectura de los apartados 1 y 2 del artículo 5 del contrato. Según esta disposición, a la fecha de la firma y en consideración del acuerdo de TexPet (posterior Chevron) de realizar el trabajo de reparación medioambiental: "El Gobierno y Petroecuador liberarán absolverán y descargarán para siempre a TexPet y a todos sus respectivos agentes, sirvientes, empleados, funcionarios, directores, representantes legales, aseguradores, abogados, indemnizadores, garantes, herederos, administradores, ejecutores administradores, ejecutores, beneficiarios, sucesores, predecesores, principales y subsidiarias (...) (a las que se denominar Exoneradas) de cualquier forma de demanda del Gobierno y Petroecuador en contra de las exoneradas por Impacto Ambiental resultante de las Operaciones del Consorcio, a excepción de aquellas relacionadas con las obligaciones del contrato, las cuales serán liberadas conforme se vaya ejecutando el trabajo de reparación ambiental de conformidad con lo previsto en el mismo (...)" (...) "Adicionalmente, el Gobierno y Petroecuador convienen en que esta liberación de demandas y compromisos no será nunca ofrecida o admitida como evidencia contra TexPet o interpretada como confesión o admisión de responsabilidad en cualquier juicio o procedimiento judicial" "El Gobierno y Petroecuador entienden por demandas cualquiera y todas las demandas, derechos de demandas, deudas, embargos, acciones y multas por causas de orden común de derecho civil o de equidad basadas en contratos o hechos dolosos, causas de acción y penalidad constitucionales, estatutarias, regulatorias (...) costos, juicios, liquidaciones y honorarios de abogados (pasado, presentes, futuros, conocidos o desconocidos) que el Gobierno o Petroecuador tengan o puedan tener en contra de cada liberación relacionada de alguna manera con la contaminación, que exista o pueda surgir directa o indirectamente de las Operaciones del Consorcio, incluyendo, pero no limitándose a las consecuencias de todos los tipos de daño que el Gobierno y Petroecuador pudieran alegar con respecto de las personas, propiedad, negocio, reputaciones, y todos los otros tipos de perjuicios que se puedan medir en términos de dinero, incluyendo, pero no limitándose a transgresiones, molestias, negligencia, responsabilidad estricta, incumplimiento de garantía, o cualquier teoría o teoría potencial de recuperación". En septiembre de 1998, PetroEcuador, PetroProduccion y TexPet firmaron un Acta final donde certificaron que TexPet había cumplido con todas sus obligaciones

CEED Número 39 Publicación actas Congreso Internacional 70 Aniversario Declaración Universal de Derechos 
Debe tenerse en cuenta que este contrato no vincula a terceros ajenos al mismo, como son los demandantes del caso Lago Agrio. Otra interpretación, la que sostiene que el Estado se comprometió a mantener la impunidad de las exoneradas incluso frente a demandas de terceros, implicaría afirmar que el Estado, para proteger a un determinado actor económico, se obligó a la vulneración de derechos humanos (como es el acceso a la justicia) presentes y futuros. En este sentido debe recordarse que en 1995 ya existían diversos procesos comenzados contra TexPet (antecesora de Chevron).

El Estado ecuatoriano habría cometido, en opinión de Chevron, una conducta "atroz e ilícita", consistente en permitir a las víctimas interponer una demanda contra ella. Posteriormente, la empresa mutaría el fondo del asunto indicando que la conducta reprobable del Ecuador fue un supuesto carácter fraudulento del procedimiento judicial del caso Lago Agrio y en la consecuente denegación de justicia que esto le supuso. En concreto, y entre otras cuestiones, la empresa afirmó que "la sentencia de primera instancia contenía partes que habían sido “escrita de manera oculta” por las víctimas”, las cuales habían sobornado a los jueces ${ }^{31}$.

Es evidente que, desde el anuncio del arbitraje, las demandantes pretendieron una intervención del poder judicial a efectos de garantizar su impunidad ${ }^{32}$. De hecho, tras la decisión de la Corte Provincial de Sucumbíos el 3 de enero de 2012, ratificando la sentencia de primera instancia, el tribunal emitió un Segundo Laudo Interino de 16 de febrero de 2012, ordenando esta vez a todas las funciones del Estado (judicial, legislativa o ejecutiva) la adopción de todas las medidas necesarias para suspender o hacer que se suspenda la ejecución y el reconocimiento dentro y fuera de Ecuador de las sentencias dictadas por la Corte Provincial de Sucumbíos, Sala Única.

bajo el acuerdo de 1995 y, por tanto, según sus disposiciones, quedaba exonerada de las responsabilidades que pudieran derivarse de una demanda interpuesta por el Gobierno o Petroecuador.

31 Memorial Suplementario, con fecha de 20 de marzo de 2012. Documento disponible en: https://www.italaw.com/sites/default/files/case-documents/ita0177.pdf

${ }^{32}$ La primera vez que el tribunal arbitral ordenó al Estado la intervención del poder judicial para paralizar el proceso Lago Agrio fue el 9 de febrero de 2011, fecha en la que el todavía no había sido dictada la sentencia de primera instancia. El Estado ecuatoriano respondió al tribunal, en una carta de 9 de enero de 2012, señalando que había hecho todo lo posible para cumplir con la Orden pero no existía (no existe) en el ordenamiento ecuatoriano una vía para cumplir semejante mandato. En este sentido, el Estado afirma que no puede violar su propia ley a efectos de satisfacer las demandas de la empresa recogidas por el tribunal. El Estado señaló además que la adopción de las medidas solicitadas por la empresa implicaría una violación de la independencia judicial y una violación directa del ordenamiento jurídico del Ecuador, en particular del artículo 168 y 126 de la Constitución ecuatoriana. Así, el Ecuador señaló que: "The Claimants have twice before requested that the Tribunal require the Republic to take additional steps to prevent enforcement of the Lago Agrio Judgment. The Republic has twice before explained that the Claimants' requested measures would compel Ecuadorian officials to violate Ecuadorian law. On both occasions this Tribunal has declined to require the Republic to violate its own law. The Republic has fully complied with the Tribunal's Interim Measures Order by taking - all measures at its disposall and notifying the Tribunal of the steps taken. Interim Measures Order”. Vid. https://www.italaw.com/sites/default/files/case-documents/ita0172.pdf 
Con la negativa del Estado ecuatoriano a intervenir el poder judicial y la negativa de los jueces a actuar en contra de la Constitución ${ }^{33}$, tras un complicado proceso, plagado de diferentes resoluciones del panel de árbitros ${ }^{34}$, llegó en agosto de 2018 el laudo sobre el fondo.

La parte operativa del fallo puede dividirse en tres apartados. En el primero, el tribunal se limita a ratificar lo ya dicho en los laudos anteriores, afirmando tanto su jurisdicción como la admisibilidad de la demanda bajo el amparo del TBI, rechazando así las objeciones del Estado. La segunda parte versa sobre el fondo e incluye la opinión del tribunal sobre los hechos; la tercera parte incluye los mandatos de actuación para la parte demandada.

En concreto, la opinión del tribunal se contiene en los apartados 10.4 a 10.12, donde se indica, entre otras cuestiones, que:

- El tribunal declara que partes importantes de la Sentencia de Lago Agrio de 14 de febrero de 2011 fueron "ghostwritten" de manera corrupta para el Juez por uno o más de los representantes de los demandantes de Lago Agrio, a cambio de la promesa de una cantidad de dinero. El tribunal considera por tanto probado que las víctimas sobornaron al juez que dictó la sentencia, algo que han negado reiteradamente las partes implicadas.

- El tribunal declara que el Estado, al emitir, hacer ejecutable, mantener la exigibilidad y ejecutar la Sentencia de Lago Agrio y, a sabiendas, facilitar su ejecución fuera del Ecuador, cometió una injusta denegación de la justicia según establecen los estándares de trato justo y equitativo y el trato requerido por el derecho internacional consuetudinario. De esta manera, el tribunal confirmaba que la no aplicación del TBI según sus propios parámetros constituye una denegación de justicia. Evidentemente, esto implica aceptar que el derecho al acceso a la justicia de la empresa que viene garantizado por el TBI es superior al acceso a la justicia de otros sujetos, aunque su derecho venga establecido en tratados internacionales de Derechos Humanos.

- El tribunal declara que el Estado de Ecuador está obligado a ofrecer una reparación completa a las empresas por esa denegación de justicia y rechaza los argumentos de defensa del Estado ${ }^{35}$. En su

\footnotetext{
${ }^{33}$ Ver Auto de 17 de febrero de 2012. Ver también Decisión de 1 de marzo de 2012, a las 16h58, de la Sala única de la Corte Provincial de Sucumbíos.

34 Todas las resoluciones pueden encontrarse en: https://www.italaw.com/cases/257
}

CEFD Número 39 Publicación actas Congreso Internacional 70 Aniversario Declaración Universal de Derechos 
opinión, la sentencia de Lago Agrio (ratificado por los pronunciamientos en apelación, casación y ante la Corte Constitucional) violó gravemente los derechos procesales fundamentales, de Chevron y es contraria a la política pública internacional, razón por la cual ninguna de sus partes debe ser reconocida o ejecutada por ningún Estado que tenga conocimiento de la denegación de justicia cometida por el Estado de Ecuador ${ }^{36}$.

- El tribunal, a efectos de "evitar dudas" declara y confirma que ni este laudo ni los anteriores laudos $\mathrm{u}$ otros escritos del procedimiento impide una demanda de ninguno de los demandantes de Lago Agrio contra Chevron que se base en un daño personal respecto de derechos individuales y que no sea una demanda colectiva (categoría en la que engloba la demanda de Lago Agrio).

Una vez sentada esta opinión, el tribunal ordena al Estado de Ecuador lo siguiente (apartado 10.13):

a) Tomar medidas inmediatas para eliminar la "ejecutabilidad" de la sentencia de Lago Agrio (desde la primera instancia a las subsiguientes).

b) Tomar medidas inmediatas para impedir que se inste el cumplimiento de parte de dicha sentencia, por cualquier medio.

c) Notificar con prontitud y por escrito del contenido de la resolución del tribunal respecto de los actos internacionalmente ilícitos, incluyendo la denegación del acceso a la justicia cometidos por el Ecuador en el caso Lago Agrio, a cualquier Estado, incluyendo su poder judicial, donde los demandantes de Lago Agrio estén intentando o puedan intentar en el presente o en el futuro la ejecución o el reconocimiento de cualquier parte de la sentencia de Lago Agrio.

Las decisiones respecto de la compensación incluyendo daños morales (de las empresas), indemnizaciones, reembolsos, pagos, intereses, etc, así como los costes del proceso se derivan a un momento posterior del proceso, Fase III, a lo largo de la cual, las partes deberán presentar subsiguientes escritos.

\footnotetext{
${ }^{35}$ Laudo 2018, párrafo 10.6.

${ }^{36}$ Laudo 2018, párrafo 10.11 .
}

CEFD Número 39 Publicación actas Congreso Internacional 70 Aniversario Declaración Universal de Derechos 128 
Como es evidente, el laudo de 2018 ordena a la República del Ecuador adoptar una decisión que implicaría intervenir el poder judicial para interferir en una causa entre particulares a efectos de defender los derechos de una de las partes (precisamente la más poderosa y la que no es un ser humano). No existe una vía jurídica en el ámbito interno que permita esta acción, por tanto, el laudo pretende obligar al Estado a adoptar una decisión ilegal, contraria al principio de independencia judicial y la separación de poderes, a la primacía de los Derechos Humanos, al reconocimiento del derecho al acceso a la justicia y a la tutela judicial efectiva, entre otros principios y derechos que están consagrados en la Constitución del Ecuador.

En relación a la colisión entre las obligaciones internacionales de la República del Ecuador en el ámbito de las inversiones y en el ámbito de los Derechos Humanos se pronunció el Experto Independiente sobre la promoción de un orden internacional democrático y equitativo, Alfred-Maurice de Zayas, en su informe de 2015, donde afirmó que los laudos interinos ordenaron la comisión de una violación del artículo 14 del Pacto Internacional de Derechos Civiles y Políticos ${ }^{37}$

Además, el relator se refirió al ordenamiento internacional señalando que, el artículo 103 de la Carta de Naciones Unidas implica que "los acuerdos de libre comercio bilaterales y multilaterales y los acuerdos de inversión que contienen disposiciones que estén en conflicto con la Carta, deben revisarse o revocarse, y deben suprimirse las disposiciones incompatibles con arreglo a la doctrina de la divisibilidad (severability)". Por último, es importante remarcar la clara obligación de jerarquización entre las obligaciones que derivan de los acuerdos de comercio e inversión y los derechos humanos que se establece en el art. 36 de la Carta de la Organización de los Estados Americanos ${ }^{38}$.

\footnotetext{
${ }^{37}$ Cuarto informe del Experto Independiente sobre la promoción de un orden internacional democrático y equitativo, Alfred-Maurice de Zayas, presentado de conformidad con lo dispuesto en la resolución 69/178. A/70/285.

38 Art. 36: Las empresas transnacionales y la inversión privada extranjera están sometidas a la legislación y a la jurisdicción de los tribunales nacionales competentes de los países receptores y a los tratados y convenios internacionales en los cuales éstos sean Parte y, además, deben ajustarse a la política de desarrollo de los países receptores. El actual marco constitucional del Ecuador y se mueve en esta línea al señalar en su artículo Art. 421 que "La aplicación de los instrumentos comerciales internacionales no menoscabará, directa o indirectamente, el derecho a la salud, el acceso a medicamentos, insumos, servicios, ni los avances científicos y tecnológicos". Además, en su artículo 416 establece los principios de las relaciones internacionales del Ecuador, que deben responder a los intereses del pueblo ecuatoriano, y que, según su apartado 12, debe fomentar "un nuevo sistema de comercio e inversión entre los Estados que se sustente en la justicia, la solidaridad, la complementariedad, la creación de mecanismos de control internacional a las corporaciones multinacionales y el establecimiento de un sistema financiero internacional, justo, transparente y equitativo. Rechaza que controversias con empresas privadas extranjeras se conviertan en conflictos entre Estados".
} 
La pregunta que suscita la lectura del caso es evidente: ¿qué papel juega el derecho internacional de los derechos humanos frente a decisiones, tratados y laudos protectores de la inversión extranjera que sitúan a las empresas como sujetos protegidos de manera preferente?. La respuesta transita, en opinión de un número creciente de voces dentro y fuera de la academia, por la apuesta por el llamado Binding Treaty.

\section{El Binding Treaty como vía para garantizar el acceso a mecanismos de justicia y reparación y la necesaria implicación de la Unión Europea.}

El 26 de junio de 2014, la hegemonía de las iniciativas basadas en textos no vinculantes dio un vuelco cuando el Consejo de Derechos Humanos de Naciones Unidas adoptó la Resolución 26/9 por la que se creó "un grupo de trabajo intergubernamental de composición abierta sobre las empresas transnacionales ETN y otras empresas con respecto a los derechos humanos, cuyo mandato es elaborar un instrumento jurídicamente vinculante para regular las actividades de las empresas transnacionales y otras empresas en el Derecho Internacional de los derechos humanos (OEIGWG)"39. La propuesta reflejó la necesidad, reiterada por diversos Estados desde la misma aprobación de los Principios Rectores, de evolucionar del ámbito de las normas basadas en la voluntariedad a un tratado internacional que asegure, entre otras cuestiones, el respeto de los derechos humanos por parte de las empresas y la garantía de los derechos de las víctimas a la justicia y la reparación. Al presentar la iniciativa, los Estados proponentes recogieron el clamor de miles de víctimas y de años de luchas, el caso Chevron y los movimientos de solidaridad fraguados durante décadas han sido y son, sin duda, uno de los ejemplos acogidos como paradigma tanto de lucha como de necesidad del llamado Binding Treaty.

La Resolución 26/9 es un documento conciso, que parte del reconocimiento de la labor realizada anteriormente, con mención expresa de las Normas de 2003, y que señala con claridad que la

\footnotetext{
39 Resolución A/HRC/RES/26/9 «Elaboración de un instrumento internacional jurídicamente vinculante sobre las empresas transnacionales y otras empresas con respecto a los derechos humanos»: adoptada con votación en el Consejo de derechos humanos $(\mathrm{CDH}) \mathrm{de}$ las Naciones Unidas el 26 de junio de 2014. Disponible en https://documents-ddsny.un.org/doc/UNDOC/GEN/G14/082/55/PDF/G1408255.pdf?OpenElement
}

CEED Número 39 Publicación actas Congreso Internacional 70 Aniversario Declaración Universal de Derechos

130 
responsabilidad principal de promover y proteger los derechos humanos implica también el reconocimiento de la responsabilidad de las empresas transnacionales ${ }^{40}$.

En este marco, la Resolución establece cuatro directrices, una respecto del contenido y tres respecto del procedimiento. Empezando por esta última cuestión, cabe señalar que la Resolución enmarcó las obligaciones de los tres primeros grupos de trabajo. Los dos primeros, que tuvieron lugar entre el 6 y el 10 de julio de $2015^{41}$, y entre el 24 y el 28 de octubre de $2016^{42}$, debían consagrarse a deliberaciones constructivas sobre el contenido, el alcance, la naturaleza y la forma del futuro instrumento internacional; en el tercero, que se celebró entre los días 23 a 27 de octubre de 2017, la Presidencia del Grupo de Trabajo presentó un documento de "elementos para el proyecto de instrumento internacional jurídicamente vinculante sobre empresas transnacionales y otras empresas con respecto a los Derechos Humanos", preparado bajo el mandato de la Resolución 26/9 y sobre la base de las dos primeras sesiones y el inicio de las negociaciones sustantivas ${ }^{\mathbf{4 3}}$. Más allá de las directrices respecto del contenido de los tres primeros grupos, la Resolución no se pronuncia sobre cómo organizar el resto de las sesiones necesarias hasta alcanzar el objetivo de la resolución: el Instrumento Vinculante. En el Informe final de la tercera sesión la Presidencia del Grupo comprometió a presentar un borrador de texto articulado en la cuarta sesión, que tuvo lugar en octubre de 2018. Este "Draf 0"44 se presentó efectivamente para ser debatido durante la cuarta sesión, recibiendo duras críticas a su contenido. El debate sobre el texto, y sus muchas carencias, continuará en la quinta sesión que está convocada para el

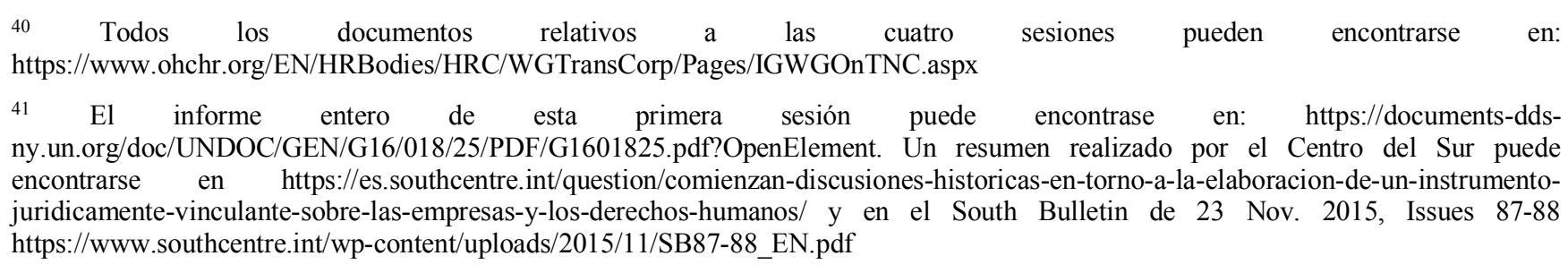
42 El informe entero de esta segunda sesión puede encontrase en: $\underline{\text { https://documents-dds- }}$ ny.un.org/doc/UNDOC/GEN/G17/000/99/PDF/G1700099.pdf?OpenElement.

43 http://www.ohchr.org/Documents/HRBodies/HRCouncil/WGTransCorp/Session3/LegallyBindingInstrumentTNCs_OBEs_SP.pdf. Sobre algunas cuestiones de este texto vid. DE SCHUTTER, Olivier. The "Elements for the draft legally binding instrument on transnational corporations and other business enterprises with respect to human rights": A Comment, 23 October 2017. Disponible en https://www.business-humanrights.org/sites/default/files/documents/ElementsTBHR-De\%20Schuttercomments23.10.2017.pdf. Consultado el 10 oct. 2017.

${ }^{44}$ Para una crítica del Draft 0 se remite al texto: GUAMÁN, A., "Del Documento de Elementos al Draft 0: apuntes jurídicos respecto del posible contenido del Proyecto de Instrumento Vinculante sobre empresas transnacionales y otras empresas con respecto a los derechos humanos". Revista de Direito Internacional. 15 - 2, pp. 85 - 115. (Brasil): Centro Universitário de Brasília, 2018. Disponible en Internet en: <https://www.publicacoes.uniceub.br/rdi/article/view/5360/3959>

CEED Número 39 Publicación actas Congreso Internacional 70 Aniversario Declaración Universal de Derechos 
mes de octubre del año 2019. En esta quinta sesión debe debatirse en torno al "Draft 1", documento que la Presidencia debe presentar recogiendo los debates de la sesión anterior.

El borrador 0 ha sido sin duda un texto polémico, que ha suscitado un profundo debate en la cuarta sesión. El Borrador, despegado de las líneas rojas marcadas por Ecuador y Sudáfrica en las sesiones anteriores, fue criticado por los aliados tradicionales de la Presidencia del grupo por haber eliminado cuestiones fundamentales como la atribución de responsabilidades a las empresas, la inclusión de las empresas públicas, la responsabilidad administrativa y de un mecanismo de control con atribuciones reales para monitorear las violaciones de derechos humanos cometidas por actores económicos parecen mínimos irrenunciables. Además, cuestiones como el enfoque de género o la especial situación de las empresas que actúan en territorios en conflicto deben ser líneas transversales, que siguen ausentes. La sociedad civil presente en las sesiones criticó de manera contundente, y casi unánime, estas cuestiones. En sentido contrario, la Unión Europea y sus aliados, en este caso contando con el bloque de países latinoamericanos con quien mantiene fuertes vínculos comerciales (Perú, Argentina, Brasil, México y Colombia) mantuvieron una durísima reticencia al texto, ya muy descafeinado, presentado por Ecuador. Así, la cuarta sesión, a diferencia de las anteriores, evidenció una soledad de la presidencia (recordamos que sigue ostentada por Ecuador) que no consiguió algo que había ocurrido en las tres primeras sesiones, la aquiescencia de la Unión Europea en la aprobación del informe final. Se plantea por tanto un proceso cuyas dificultadas suben año tras año.

Como es evidente, la cuestión jurídica en este proceso hacia el Binding Treaty es trascendida y traspasada en cada uno de los artículos del futuro tratado por un sinfín de aristas políticas que además varían de sesión en sesión. Las cuestiones más debatidas siguen siendo las siguientes: el ámbito de aplicación del Instrumento; la atribución de responsabilidades directas a las empresas; el acceso a la justicia, los mecanismos jurisdiccionales en el ámbito estatal y la exigencia de responsabilidad por actos cometidos en otro Estado; la relación entre el Derecho Internacional de los Derechos Humanos y los tratados de inversión y finalmente el mecanismo de control a nivel internacional que debe establecerse en el IJV. Cada uno de estos temas conlleva, como es evidente, una serie de intereses políticos que han dividido a los países participantes en dos grandes bloques, los cuales han evolucionado a lo largo de las negociaciones.

El cambio trascendental en la línea seguida por el gobierno de Ecuador en los últimos meses; el reposicionamiento de las alianzas geopolíticas regionales e internacionales dentro y fuera del 
GRULAG; la presión creciente de Estados Unidos hacia países pequeños o, en sentido inverso, la actividad creciente de la sociedad civil y otros actores como el Parlamento Europeo sobre los estados que componen la Unión pueden ser factores que marque desde el inicio el contenido de la quinta sesión.

Sea cual sea el desenlace del proceso, lo que es indudable es que el proceso de la 26/9 ha despertado una enorme expectativa entre la sociedad civil global y entre numerosos Estados, trascendiendo igualmente a otros importantes actores como los sindicatos y la academia. En este sentido, los abundantes debates generados en los últimos meses sobre la posibilidad y la necesidad del Binding Treaty, apoyados en buena medida en la urgencia que evidencia el caso Chevron, son sin duda una puerta para la evolución y la transformación de los mecanismos de garantía y la reparación de los derechos humanos, que no puede desaprovecharse.

CEFD Número 39 Publicación actas Congreso Internacional 70 Aniversario Declaración Universal de Derechos 
CANTÚ, H. (Coord.); Derechos Humanos y Empresas: Reflexiones desde América Latina. Costa Rica. Instituto Interamericano de Derechos Humanos, 2017.

CECEÑA, A.E., ORNELAS, R. (Coords.); Chevron. Paradigma de la catástrofe civilizatoria, Siglo XXI, México, UNAM, Instituto de Investigaciones Económicas, 2017.

DEVA, S., BILCHITZ, D. (Eds.); Building a Treaty on Business and Human Rights. Context and Contours. Cambridge. Cambridge University Press. 2017.

DEVA, S., BILCHITZ, D., (Eds.); Human Rights Obligations of Business: Beyond the Corporate Responsibility to Respect?. Cambridge. Cambridge University Press. 2013.

DOLZER, R. Y STEVENS, M., Bilateral Investment Treaties, Nijhoff, Dordrecht, 1995.

EBERHARDT, P., Investment Protection at a Crossroads. The TTIP and the Future of International Investment Law. Friedrich Eber Stiftung, 2014. http://library.fes.de/pdf-files/iez/global/10875.pdf.

ESTEVE, J.E., "Los Principios Rectores sobre las empresas transnacionales y los derechos humanos en el marco de las Naciones Unidas para «proteger, respetar y remediar»: ¿hacia la responsabilidad de las corporaciones o la complacencia institucional?", Anuario Español de derecho internacional, N 27, 2011.

ESTEVE, J.E., "Planes de Acción Nacional sobre empresas y derechos humanos: la imperiosa complementariedad con normas vinculantes. Referencia al plan español”. Anuario español de derecho internacional, $\mathrm{N}^{\mathrm{o}} 34,2018$.

GUAMÁN, A., "Del Documento de Elementos al Draft 0: apuntes jurídicos respecto del posible contenido del Proyecto de Instrumento Vinculante sobre empresas transnacionales y otras empresas con respecto a los derechos humanos". Revista de Direito Internacional. 15 - 2, pp. 85 - 115. (Brasil): Centro Universitário de Brasília, 2018. Disponible en Internet en: $<$ https://www.publicacoes.uniceub.br/rdi/article/view/5360/3959>

GUAMÁN, A., GONZÁLEZ, G.; Empresas Transnacionales y Derechos Humanos, Albacete, Bomarzo, 2018.

HERNÁNDEZ ZUBIZARRETA, J.; Las empresas transnacionales frente a los derechos humanos. Historia de una asimetría normativa. Vitoria, Egoa. 2009

KRAJEWSKI, M., Modalities for investment protection and Investor-State Dispute Settlement (ISDS) in TTIP from a trade union perspective, Friedrich-Alexander-Universität Erlangen-Nürnberg, 2015. Disponible en http://library.fes.de/pdf-files/bueros/bruessel/11044.pdf;

LYON-CAEN, A., SACHS, T., "The Responsibility of Multinational Entreprise. A constitutionalization process", en ROBÉ, J.-P., LYON-CAEN, A., VERNAC, S., (dir.), Multinationals and the constitutionalisation of the World Power System, Routlege, 2016. 
MARULLO, Ma CH. Y ZAMORA CABOT, F.J. (eds.), Empresas y Derechos Humanos, Scientifica, Nápoles, 2018.

PIGRAU, A., "The Texaco-Chevron case in Ecuador: Law and justice in the age of globalization", Revista catalana de dret ambiental, Vol. V Núm. 1 (2014): 1 - 43.

PRIETO, J., ESPINOZA, G., “A binding treaty on corporate responsibility: a global solution to address the problem of corporate impunity. Lessons learned from Aguinda vs Chevron", en HOMA Publica: International Journal on Human Rigths and Business, N², 2017

Procuraduría General del Estado, Caso Chevron: Defensa del Ecuador frente al uso indebido del arbitraje de inversión, Quito, 2015. Disponible http://www.pge.gob.ec/images/stories/boletines/lanzamientolibrochevron/libro_Caso_CHEVRON.pdf

SKINNER, G., et alt., «El Tercer Pilar: El acceso efectivo a los recursos judiciales para las víctimas de las violaciones de los derechos humanos cometidas por empresas transnacionales», International Corporate Accountability Roundtable, icar, core y European Coalition for Corporate Justice, ECCJ, 2013

SORNARAJAH, M., The international Law of foreing investment, Cambridge, Cambridge University Press, 2004.

VICENTE BLANCO, D. J., "El conflicto histórico por el régimen de la inversión extranjera directa", en VICENTE BLANCO, D. J., (Ed.) La libertad del dinero, Valencia, Germanía, 2003.

ZAMORA CABOT, F.J., "Las empresas multinacionales y su Responsabilidad en materia de derechos Humanos: una visión de conjunto" en Working Papers “El Tiempo de los Derechos”, N6, 2013. 